\title{
SOME NOTEWORTHY PROPERTIES OF ZERO DIVISORS IN INFINITE RINGS II
}

\author{
HOWARD E. BELL* ${ }^{*}$ and ABRAHAM A. KLEIN
}

In [3] we have considered four subsets of zero divisors of an infinite ring $R$ which is not a domain: $D=D(R)$ - the set of all zero divisors; $T=T(R)-$ the set of two-sided zero divisors; $S=S(R)$ - the set of zero divisors with nonzero two-sided annihilator; and $N=N(R)$ - the set of nilpotent elements. Our main interest was in the sets $S \backslash N, T \backslash S$ and $D \backslash T$. We have seen that these sets are power closed and root closed, where a subset of a ring is said to be root closed if whenever it contains a positive power of an element, it also contains the element itself. The main results of [3] were: If $S \neq N$ then $S \backslash N$ is infinite; and if $N$ is infinite, then each of the sets $T \backslash S$ and $D \backslash T$ is infinite provided it is nonempty. We have also constructed examples showing that among the eight formal conditions obtainable by choosing sequences of equalities and proper inclusions in $D \supseteq T \supseteq S \supseteq N$, all except perhaps $D \neq T \neq S=N$ can be satisfied. In the present paper we construct a ring satisfying $D \neq T \neq S=N$. The main results of the paper refer to a fifth set of zero divisors which is located between $S$ and $N$. We first consider the subset of $S$ of elements for which the left and right annihilator coincide, and we denote this set by $S_{1}$. For example, all zero divisors belonging to the center are in $S_{1}$. The set $S_{1}$ need not contain $N$, so we prefer to consider the set of elements radical over $S_{1}$; and we denote it by $W$. We clearly have $S \supseteq W \supseteq N$. We prove that $W$ is infinite, and if $W \neq N$, then $W \backslash N$ is infinite. If $S \neq W$, then $S \backslash W$ may be finite; but it is infinite when $N$ is infinite or when $R$ has 1 . As regards $S_{1}$, we prove that it is infinite when $N$ is finite; and if $R$ has 1 and $S_{1} \backslash N$ is nonempty, then $S_{1} \backslash N$ is infinite.

We close the paper with the result that $S$ has the same cardinal number as $R$. This improves the similar result for $T$ which was proved by Lanski [4].

\footnotetext{
* Supported by the Natural Sciences and Engineering Research Council of Canada, Grant No. 3961.

Received June 17, 1996.
} 
1. A ring with $D \neq T \neq S=N$.

We first construct a ring with 1 for which $D=T \neq S=N$. We start with the ring of integral polynomials $\mathrm{Z}[t]$ and its subring (ideal) $P=t \mathrm{Z}[t]$. Let $V$ be the zero ring on the additive group of $P$. Consider $V$ as a $P$-module (left and right) under the multiplication of elements of $P$. Let

$$
R=\left[\begin{array}{ll}
P & V \\
V & 0
\end{array}\right]
$$

with the obvious multiplication. We have $D=T \neq S=N$ as in Example 5 of [3]. In that example, the property $D=T \neq S=N$ is lost when 1 is adjoined, but here this property is preserved. Indeed, if $R^{1}$ denotes the ring obtained from $R$ by adjoining 1 , then the elements of $R^{1} \backslash R$ are easily seen to be regular in $R^{1}$ and therefore the sets $D, T, S, N$ remain unchanged. Note that the same idea may be used to construct rings with 1 satisfying the other conditions considered in [3].

The desired example is the ring $Q=R^{1}[x ; \sigma]$ of skew left polynomials in $x$, where $x a=\sigma(a) x$ for $a \in R^{1}$ and $\sigma$ is the endomorphism of $R^{1}$ sending 1 to 1 and $\left[\begin{array}{cc}p & v^{\prime} \\ v & 0\end{array}\right]$ to $\left[\begin{array}{cc}p & v^{\prime} \\ 0 & 0\end{array}\right]$. We proceed to show that $D(Q) \neq T(Q) \neq$ $S(Q)=N(Q)$.

We shall use the same notation for annihilators as in [3]. Let $J=\left[\begin{array}{ll}0 & 0 \\ V & 0\end{array}\right]$ and $J^{\prime}=\left[\begin{array}{ll}0 & V \\ 0 & 0\end{array}\right]$, and note that $J=\operatorname{ker} \sigma$. We have $x \in D(Q) \backslash T(Q)$, since $A_{\ell}(x)=0$ and $A_{r}(x) \supset J$. If $a \in T(R) \backslash N(R)$, then we clearly have $A_{\ell}(a)=J^{\prime}[x ; \sigma]$ and $A_{r}(a)=J[x ; \sigma]$, so $a \in T(Q) \backslash S(Q)$, since $J \cap J^{\prime}=\{0\}$. It remains to show that $S(Q)=N(Q)$.

Any $f(x) \in Q$ has a unique decomposition $f(x)=f_{0}(x)+f_{1}(x)$ with $f_{0}(x) \in R[x ; \sigma]$ and $f_{1}(x) \in \mathbf{Z}[x]$. If $g(x) \in Q$, then $(f g)_{1}(x)=f_{1}(x) g_{1}(x)$, so $\left(f^{n}\right)_{1}(x)=\left(f_{1}(x)\right)^{n}$; therefore, if $f(x) \in N(Q)$, then $f_{1}(x)=0$. Thus if $f(x) \in N(Q)$, we may identify $f(x)=\Sigma\left[\begin{array}{cc}p_{i} & v_{i}^{\prime} \\ v_{i} & 0\end{array}\right] x^{i}$ with $\left[\begin{array}{cc}\Sigma p_{i} x^{i} & \Sigma v_{i}^{\prime} x^{i} \\ \Sigma v_{i} x^{i} & 0\end{array}\right]$. The $(1,1)$-entry of $f^{n}(x)$ is $\left(\Sigma p_{i} x^{i}\right)^{n}$; and since $f(x)$ is nilpotent, we have $\Sigma p_{i} x^{i}=0$, so that $p_{i}=0$ for all $i$. Thus $f(x)$ is a polynomial with coefficients in $N(R)$; and since the square of any such polynomial is 0 , we can conclude that $N(Q)=N(R)[x ; \sigma]$.

To prove $S(Q)=N(Q)$, we take $f(x) \in T(Q) \backslash N(Q)$ and show that $A_{\ell}(f(x))=J^{\prime}[x ; \sigma]$ and $A_{r}(f(x))=J[x ; \sigma]$, so that $f(x) \notin S(Q)$. Our argument will make use of the following lemma: 
Lemma 1. If $f(x), g(x) \in Q \backslash\{0\}$ and $g(x) f(x)=0$, then either $f_{1}(x)=0$ or $A_{\ell}(f(x))=0$.

Proof. Assume $f_{1}(x)=\Sigma m_{i} x^{i} \neq 0$, and let $g(x) f(x)=0$ with $g(x)=$ $g_{0}(x)+g_{1}(x), g_{0}(x) \in R[x ; \sigma], g_{1}(x) \in \mathrm{Z}[x]$. Then $g_{1}(x) f_{1}(x)=0$, so $g_{1}(x)=0$ and $g(x)=\Sigma\left[\begin{array}{cc}b_{j} & w_{j}^{\prime} \\ w_{j} & 0\end{array}\right] x^{j}$. We have

$$
\begin{gathered}
0=g(x) f(x)= \\
{\left[\begin{array}{cc}
b_{0} & w_{0}^{\prime} \\
w_{0} & 0
\end{array}\right] \Sigma\left[\begin{array}{cc}
a_{i}+m_{i} & v_{i}^{\prime} \\
v_{i} & m_{i}
\end{array}\right] x^{i}+\Sigma_{j \geq 1}\left[\begin{array}{cc}
b_{j} & w_{j}^{\prime} \\
w_{j} & 0
\end{array}\right] x^{j} \Sigma\left[\begin{array}{cc}
a_{i}+m_{i} & v_{i}^{\prime} \\
v_{i} & m_{i}
\end{array}\right] x^{i} ;}
\end{gathered}
$$

and since for $j \geq 1 \quad x^{j}\left[\begin{array}{cc}a_{i} & v_{i}^{\prime} \\ v_{i} & 0\end{array}\right]=\left[\begin{array}{cc}a_{i} & v_{i}^{\prime} \\ 0 & 0\end{array}\right] x^{j}=x^{j}\left[\begin{array}{cc}a_{i} & v_{i}^{\prime} \\ 0 & 0\end{array}\right]$, we obtain

$$
\begin{aligned}
0 & =\left[\begin{array}{cc}
b_{0} & w_{0}^{\prime} \\
w_{0} & 0
\end{array}\right]\left[\begin{array}{cc}
\Sigma\left(a_{i}+m_{i}\right) x^{i} & \Sigma v_{i}^{\prime} x^{i} \\
\Sigma v_{i} x^{i} & \Sigma m_{i} x^{i}
\end{array}\right] \\
& +\left[\begin{array}{cc}
\Sigma_{j \geq 1} b_{j} x^{j} & \Sigma_{j \geq 1} w_{j}^{\prime} x^{j} \\
\Sigma_{j \geq 1} w_{j} x^{j} & 0
\end{array}\right]\left[\begin{array}{cc}
\Sigma\left(a_{i}+m_{i}\right) x^{i} & \Sigma v_{i}^{\prime} x^{i} \\
0 & \Sigma m_{i} x^{i}
\end{array}\right] .
\end{aligned}
$$

The $(1,1)$-entry is $\Sigma b_{j} x^{j} \Sigma\left(a_{i}+m_{i}\right) x^{i}=0$; and since $\Sigma\left(a_{i}+m_{i}\right) x^{i} \neq 0$, we get $b_{j}=0$ for all $j$. In a similar way, considering the $(2,1)$-entry, we get $w_{j}=0$ for all $j$, hence $w_{j}^{\prime}=0$ for all $j$ since $\Sigma m_{i} x^{i} \neq 0$. Thus, $A_{\ell}(f(x))=0$; and the lemma is established.

Returning to our main argument, let $f(x) \in T(Q) \backslash N(Q)$; and note that by Lemma $1, f_{1}(x)=0$. Let $g(x) \in A_{r}(f(x))$. Since $0 \neq f(x) \in A_{\ell}(g(x))$, Lemma 1 gives $g_{1}(x)=0$; therefore,

$$
\begin{aligned}
0 & =f(x) g(x)=\left[\begin{array}{cc}
a_{0} & v_{0}^{\prime} \\
v_{0} & 0
\end{array}\right]\left[\begin{array}{cc}
\Sigma b_{j} x^{j} & \Sigma w_{j}^{\prime} x^{j} \\
\Sigma w_{j} x^{j} & 0
\end{array}\right] \\
& +\left[\begin{array}{cc}
\Sigma_{i \geq 1} a_{i} x^{i} & \Sigma_{i \geq 1} v_{i}^{\prime} x^{i} \\
\Sigma_{i \geq 1} v_{i} x^{i} & 0
\end{array}\right]\left[\begin{array}{cc}
\Sigma b_{j} x^{j} & \Sigma w_{j}^{\prime} x^{j} \\
0 & 0
\end{array}\right] .
\end{aligned}
$$

Then $\Sigma a_{i} x^{i} \Sigma b_{j} x^{j}=0$; but $\Sigma a_{i} x^{i} \neq 0$ since $f(x) \notin N(Q)$, so $b_{j}=0$ for all $j$. Similarly we get $w_{j}^{\prime}=0$ for all $j$, so $g(x) \in J[x ; \sigma]$ and therefore $A_{r}(f(x))=J[x ; \sigma]$.

Now consider $g(x) \in A_{\ell}(f(x)), g_{0}(x)=\Sigma\left[\begin{array}{cc}b_{j} & w_{j}^{\prime} \\ w_{j} & 0\end{array}\right] x^{j}, g_{1}(x)=\Sigma n_{j} x^{j}$. Calculating the product $g(x) f(x)$, we obtain $\Sigma\left(b_{j}+n_{j}\right) x^{j} \Sigma a_{i} x^{i}=0$, so $\Sigma\left(b_{j}+n_{j}\right) x^{j}=0$ and $b_{j}=0, n_{j}=0$ for all $j$. Similarly we get $w_{j}=0$ for all $j$, 
so $g(x) \in J^{\prime}[x ; \sigma]$ and therefore $A_{\ell}(f(x))=J^{\prime}[x ; \sigma]$. Thus $S(Q)=N(Q)$, hence for the ring $Q$ we have $D \neq T \neq S=N$.

\section{The sets $S_{1}$ and $W$.}

Consider the following set of zero divisors:

$$
S_{1}=\left\{a \in S \mid A_{\ell}(a)=A_{r}(a)\right\} .
$$

For $a \in S_{1}, A(a)=A_{\ell}(a)=A_{r}(a)$ is a nonzero ideal, so $S_{1}=\{0\}$ if the ring is prime. It is easy to show that $S_{1}$ is power closed, however $S_{1}$ is not in general root closed. For example, if $R$ is a prime ring which is not a domain, $R$ has nonzero nilpotent elements but $S_{1}=\{0\}$.

Given a subset $V$ of a ring $R$, one may define its root closure by

$$
\bar{V}=\left\{a \in R \mid a^{n} \in V \text { for some } n \geq 1\right\} .
$$

Clearly $\bar{V}$ is root closed, it contains $V$, and it is the minimal root closed subset of $R$ containing $V$. Moreover, if $V$ is power closed, then $\bar{V}$ is power closed. Note that $N=\overline{\{0\}}$.

Now let $W=\bar{S}_{1}$. Since $S$ is root closed and $S \supseteq S_{1} \supseteq\{0\}$, we have $S \supseteq W \supseteq N$; and $W=N$ if and only if $S_{1} \subseteq N$. We may have $S \underset{\neq}{\supset} \underset{\neq}{\supset} N-$ for example in $R=M_{2}(Q) \oplus \mathbf{Z}_{6}$, where $\left(e_{11}, 0\right) \in S \backslash W$ and $(0,1) \in W \backslash N$. In any ring, $W$ is power closed, since $S_{1}$ is power closed.

Recall that we consider only infinite rings with $D \neq\{0\}$. As mentioned in [3], $S$ is infinite, and this result is improved as follows.

Theorem 1. If $R$ is any infinite ring with $D \neq\{0\}$, then $W$ is infinite.

Proof. If $N$ is infinite, we are done, since $W \supseteq N$. Let $N$ be finite and $R$ semiprime. Then, by [2, Cor. 5], $R=R_{1} \oplus R_{2}$ where $R_{1}$ is reduced and $R_{2}$ is finite; and clearly $R_{1}$ is infinite. If $R_{2} \neq\{0\}$, then $S_{1} \supseteq R_{1}$; and if $R_{2}=\{0\}$, then $S_{1}=D$. Thus $S_{1}$ is infinite and so is $W$.

Now assume $N$ is finite and the prime radical $\mathscr{P}(R)$ is nonzero, and let $\bar{R}=R / \mathscr{P}(R)$. Then, again by [2, Cor. 5], $\bar{R}$ is a direct sum of a reduced ring and a finite ring; and we denote their inverse images in $R$ by $R_{1}$ and $R_{2}$ respectively. We have $\bar{R}=\bar{R}_{1} \oplus \bar{R}_{2}$, and $R_{1}$ has finite index in $R$, since $\bar{R}_{1}$ has finite index in $\bar{R}$. By [3, Lemma 1], $A=A(\mathscr{P}(R))$ is an ideal of $R$ of finite index, since $\mathscr{P}(R)$ is a finite ideal. It follows that $A_{1}=A \cap R_{1}$ has finite index, so $A_{1}$ is infinite; and we proceed to prove that $A_{1} \subseteq W$.

For any two elements $u, v$ of a reduced ring, it is easy to see that if one of the products $u v, v u, u^{2} v, v u^{2}$ is 0 , so are all others. Since $\bar{R}_{1}$ is reduced and $\bar{R}=\bar{R}_{1} \oplus \bar{R}_{2}$, we get for $x \in R_{1}, y \in R$ the result that if one of the products $\bar{x} \bar{y}, \bar{y} \bar{x}, \bar{x}^{2} \bar{y}, \bar{y} \bar{x}^{2}$ is $\overline{0}$, so are all others; otherwise put, if one of the products 
$x y, y x, x^{2} y, y x^{2}$ is in $\mathscr{P}(R)$, so are all others. It follows for $x \in A_{1}, y \in R$ that $x^{2} y=0$ implies $y x \in \mathscr{P}(R)$, hence $y x^{2}=0$; and similarly $y x^{2}=0$ implies $x^{2} y=0$. We have shown that $A_{\ell}\left(x^{2}\right)=A_{r}\left(x^{2}\right)$,so $x^{2} \in S_{1}$ and $x \in W$. Thus $A_{1} \subseteq W$, as we wished to prove.

Corollary. If $R$ is an infinite ring with $D \neq\{0\}$ and $N$ finite, then $S_{1}$ is infinite.

Proof. The case when $R$ is semiprime is considered at the beginning of the proof of Theorem 1.

If $R$ is not semiprime, we have seen in the proof of Theorem 1 that $x^{2} \in S_{1}$ if $x \in A_{1}$. Now $A_{1}$ is an infinite subring of $R_{1}$, so $\bar{A}_{1}$ is an infinite reduced ring. Assuming $S_{1}$ is finite, we have that $\left\{x^{2} \mid x \in A_{1}\right\}$ is finite, so $\left\{\bar{x}^{2} \mid \bar{x} \in \bar{A}_{1}\right\}$ is finite. But by [1, Th. 4.1] it follows that $\bar{A}_{1}$ is finite -- a contradiction.

\section{The sets $W \backslash N$ and $S_{1} \backslash N$.}

Since $N, W, S$ are power closed and root closed, so are $W \backslash N$ and $S \backslash W$.

From now on, the results will be stated without saying that it is assumed that $R$ is infinite and $D \neq\{0\}$. The center of $R$ is denoted by $Z$.

Lemma 2. If $e \in W$ is an idempotent, then $e \in Z$.

Proof. We have $e=e^{2}=\cdots$, so $e \in S_{1}$ and $A_{\ell}(e)=A_{r}(e)$. Since $e(x e-e x e)=0$ and $(e x-e x e) e=0$, we obtain $x e-e x e=0$ and $e x-e x e=0$, so $x e=e x$.

THeOREm 2. If $W \backslash N$ is nonempty, then it is infinite.

Proof. If $N$ is finite, the result follows by Theorem 1.

Let $N$ be infinite and $a \in W \backslash N$. Then $a^{m} \in W \backslash N$ for any $m \geq 1$, so if $a$ has infinitely many distinct powers, we are done. Otherwise some power of $a$ is a nonzero idempotent $e$, and $e \in Z$ by Lemma 2 .

Now $n e \in Z$ for any integer $n$, hence $n e \in W$; thus, if $e$ has infinite additive order, we are done. Assume $k e=0$ for some $k>1$. Since $N$ is infinite, there are infinitely many elements squaring to $0[2$, Th. 6]; and for each such element $u,(e+u)^{k}=e+k e u=e$. Therefore the infinite set $\left\{e+u \mid u^{2}=0\right\}$ is contained in $W \backslash N$.

THEOREM 3. If $R$ has 1 and $S_{1} \backslash N$ is nonempty, then $S_{1} \backslash N$ is infinite.

Proof. When $N$ is finite, the result follows from the corollary in the previous section. When $N$ is infinite we follow the arguments given in the proof of Theorem 2, starting with $a \in S_{1} \backslash N$ and obtaining an idempotent $e \in S_{1} \backslash N$ 
with $k e=0$ for some $k>1$. By [2, Th. 6] $R$ has an infinite zero subring $U$, and either $e U$ or $(1-e) U$ is infinite. Since $R$ has $1,1-e$ is an idempotent belonging to $S_{1} \backslash N$, so we may assume without loss that $e U$ is infinite. For $u \in U$ we have $(e+e u)^{k}=e$, so $e+e u \notin N$ and $A_{\ell}(e+e u)=A_{\ell}(e)$. Similarly $A_{r}(e+e u)=A_{r}(e)$, so $e+e u \in S_{1}$ since $e \in S_{1}$. Thus $S_{1} \backslash N$ contains the infinite set $e+e U$.

\section{The set $S \backslash W$.}

We start with an example showing that $S \backslash W$ may be finite and nonempty.

Let $\mathrm{Z}_{p}$ be the field of $p$ elements, let $C_{p}$ be the zero ring on the cyclic group of order $p$ with generator $u$, and let $J$ be an infinite domain. Let $R=\mathrm{Z}_{p} \times C_{p} \times J$ with addition as in $\mathrm{Z}_{p} \oplus C_{p} \oplus J$ and multiplication determined by $e u=u$, $u e=0, e J=J e=0$ and $u J=J u=0$. This gives a ring structure on $R$. We have $D=R$ since $u R=0 ;$ and $T=S=$ $\left(\mathrm{Z}_{p} \oplus C_{p}\right) \cup\left(C_{p} \oplus J\right)$. Now if $0 \neq a \in J$, then $A_{\ell}(a)=A_{r}(a)=\mathrm{Z}_{p} \oplus C_{p}$, so $J \subseteq S_{1} \subseteq W$; and since $C_{p}^{2}=0$ we have $C_{p} \oplus J \subseteq W$, and it follows easily that $C_{p} \oplus J=W$. Thus $S \backslash W=\left(Z_{p} \backslash\{0\}\right) \oplus C_{p}$ is finite and nonempty.

In the previous example, $N$ is finite. We now proceed to consider $S \backslash W$ when $N$ is infinite. We start with a simple result, which holds in arbitrary rings.

Lemma 3. (1) Let e be a noncentral idempotent. Then either there is an element $v \neq 0$ satisfying $e v=v$, ve $=v^{2}=0$, or there is an element $u \neq 0$ satisfying $u e=u$,eu $=u^{2}=0$.

(2) If for an element $v \neq 0(u \neq 0)$ there is an element a satisfying $a v=v, v a=0(u a=u, a u=0)$, then $a \in D \backslash W$.

Proof. (1) Since $e \notin Z$, we have $e R(1-e) \neq 0$ or $(1-e) R e \neq 0$. If $e R(1-e) \neq 0$, take $v \neq 0$ in $e R(1-e)$; otherwise take $u \neq 0$ in $(1-e) R e$.

(2) By symmetry it suffices to prove the result for $v$. We have $a \in D$ since $v \neq 0$; and $a^{k} v=v, v a^{k}=0$ for any $k \geq 1$, so $a \notin W$.

THEOREM 4. If $N$ is infinite and $S \backslash W$ is nonempty, then $S \backslash W$ is infinite.

Proof. As in the proof of Theorem 2, we may assume there is an idempotent $e \in S \backslash W$. Then $e \notin Z$; and applying Lemma 3, we may assume there is an element $v \neq 0$ satisfying $e v=v, v e=v^{2}=0$. As in the proof of Theorem 3 , we let $U$ be an infinite zero subring; and we consider separately the two cases: (1) eUe infinite, (2) eUe finite.

In case (1) $e U$ is infinite; and if $e u \in e U \backslash N, u \in U$, then for $k \geq 1,(e u)^{k} u=$ $0 \neq u(e u)^{k}$, so $e u \notin W$. Since $e \in S, A(e) \neq 0$; and if $0 \neq b \in A(e)$, then $b \in A(e u)$ when $u b=0$ and $u b \in A(e u)$ when $u b \neq 0$, so $e u \in S \backslash W$. Thus we 
may assume $e U \backslash N$ is finite and therefore $e U \cap N$ is infinite. It follows that there are infinitely many elements of the form $e+e u e$ where $e u \in e U \cap N$. Clearly they are all in $S$, and we prove that none is in $W$. We have $v(e+e u e)^{k}=0$ for all $k \geq 1$. On the other hand, if $(e+e u e)^{k} v=0$ for some $k$, then since $e$ commutes with eue and $e v=v$, we have

$$
0=v+\left(\sum_{i=1}^{k}\left(\begin{array}{c}
k \\
i
\end{array}\right)(e u)^{i}\right) v .
$$
Since $e u \in N, \sum_{i=1}^{k}\left(\begin{array}{l}k \\ i\end{array}\right)(e u)^{i} \in N$; and it follows from (*) that $v=0$-- a contra-
diction.

In case (2), if $e U$ and $U e$ are finite, then each of $A_{\ell}(e) \cap U$ and $A_{r}(e) \cap U$ has finite index in $(U,+)$ and so does $A(e) \cap U$, which is therefore infinite. It follows that either $v(A(e) \cap U)$ is infinite or $A(e) \cap U \cap A_{r}(v)$ is infinite. If $v(A(e) \cap U)$ is infinite, we have infinitely many elements of the form $e+v u$ where $u \in A(e) \cap U$. For each such element $v(e+v u)=0$ and $(e+v u) v=v$, so $e+v u \notin W$; moreover, $e+v u \in S$, since $u \in A(e+v u)$. If $A(e) \cap U \cap A_{r}(v)$ is infinite, then for any $u$ in this set we have $(e+u) v=v$ and $v(e+u)=0$, so $e+u \notin W$; and also $u \in A(e+u)$, so $e+u \in S \backslash W$.

It remains to consider case (2) with $e U$ infinite or $U e$ infinite. If $e U$ is infinite, then $e U \cap A_{\ell}(e)$ is infinite, since $e U e$ is finite. For any nonzero element $e u \in e U \cap A_{\ell}(e), u \in U$, we have $e u(e+e u)=0$ and $(e+e u) e u=e u$, so $e+e u \notin W$. If $0 \neq b \in A(e)$, then $0 \neq b-u b \in A(e+e u)$, hence $e+e u \in S$. In a similar way, when $U e$ is infinite, the infinite set $e+U e \cap A_{r}(e)$ is contained in $S \backslash W$. This completes the proof of Theorem 4.

We have seen that $S \backslash W$ may be nonempty and finite. However, we have

THEOREM 5. If $R$ has 1 and $S \neq W$, then $S \backslash W$ is infinite.

Proof. We may assume $N$ is finite and, as in the proof of Theorem 4, let $e$ be an idempotent in $S \backslash W$ and $v$ a nonzero element satisfying $e v=v$, $v e=v^{2}=0$. Using the notation as in the proof of Theorem 1, we have $\bar{R}=R / \mathscr{P}(R)=\bar{R}_{1} \oplus \bar{R}_{2}$, where $\bar{R}_{1}$ is infinite and reduced and $\bar{R}_{2}$ is finite. For $\bar{x} \in \bar{R}$ write $\bar{x}=\bar{x}_{1}+\bar{x}_{2}, \bar{x}_{i} \in \bar{R}_{i}$. Letting $\bar{e}=\bar{e}_{1}+\bar{e}_{2}$, we observe that $\bar{e}_{1}$ is a central idempotent in $\bar{R}_{1}$ and $[\bar{e}, \bar{x}]=\left[\bar{e}_{2}, \bar{x}_{2}\right]$; and since $\mathscr{P}(R)$ and $\bar{R}_{2}$ are finite, we see that there are only finitely many commutators of the form $e x-x e, x \in R$. Therefore $C=C_{R}(e)$ is of finite index in $R$, hence infinite.

Assume $e C$ is infinite. We have $e C v \subseteq N$, so $e C v$ is finite, and hence $e C \cap A_{\ell}(v)$ is infinite. For any $u \in e C \cap A_{\ell}(v),(e+u) v=v$ and $v(e+u)=0$, so $e+u \notin W$; and since $u \in e C$ and $C$ commutes with $e$, we see that $A(e) \subseteq A(e+u)$, so $e+u \in S \backslash W$.

If $e C$ is finite, then $(1-e) C$ is infinite; also, $1-e$ is an idempotent not in 
$W$ and $(1-e) v=0, v(1-e)=v$. Thus we may replace $e$ by $1-e$ and proceed as above.

If $D \backslash W$ is nonempty, then at least one of the sets $S \backslash W, T \backslash S, D \backslash T$ is nonempty; hence by Theorem 4 and [3, Th. 4, Th. 5], if $N$ is infinite, then $D \backslash W$ is infinite. This conclusion may be established directly without assuming that $N$ is infinite.

TheORem 6. If $D \backslash W$ is nonempty, then it is infinite.

Proof. As before, we may assume there is an idempotent $e$ in $D \backslash W$ and an element $v \neq 0$ satisfying $e v=v, v e=v^{2}=0$.

Let $K$ be the kernel of the map $a \mapsto v a v$ from $R$ onto $v R v$; and note that $v R v, K v, v K \subseteq A(v)$. Thus, if one of $v R v, K v, v K$ is infinite, then $A(v)$ is infinite. On the other hand, if all three are finite, then $K$ is infinite and $K \cap A_{\ell}(v)$ and $K \cap A_{r}(v)$ have finite index in $K$, in which case $K \cap A(v)$ has finite index in $K$. Thus, in any event $A(v)$ is infinite.

Now for $u \in A(v)$ we have $(e+u) v=v$ and $v(e+u)=0$, so the infinite set $e+A(v)$ is contained in $D \backslash W$.

Note that the example given at the beginning of this section shows that in the above theorem $D$ cannot be replaced by $T$.

We close the paper by improving a result of Lanski [4, Th. 6], which states that the cardinal number of $T$ equals that of $R$. Our result is:

Theorem 7. $\operatorname{Card}(S)=\operatorname{Card}(R)$.

Proof. Simply repeat Lanski's proof with $T$ replaced by $S$. For the convenience of the reader it is suggested to replace $S, W$ appearing in Lanski's proof by $N, K$ respectively.

\section{REFERENCES}

1. H. E. Bell and A. A. Klein, Ideals contained in subrings, Houston J. Math. 24 (1998), 1-8.

2. A. A. Klein and H. E. Bell, Rings withfinitely many nilpotent elements, Comm. Algebra 22 (1994), 349-354.

3. A. A. Klein and H. E. Bell, Some noteworthy properties of zero divisors in infinite rings, Math. Scand. 75 (1994), 59-66.

4. C. Lanski, Rings with few nilpotents, Houston J. Math. 18 (1992), 577-590.

DEPARTMENT OF MATHEMATICS

BROCK UNIVERSITY

ST. CATHARINES, ONTARIO

CANADA L2S 3A1

e-mail: hbell@spartan.ac.brocku.ca
SCHOOL OF MATHEMATICAL SCIENCES SACKLER FACULTY OF EXACT SCIENCES TEL AVIV UNIVERSITY

TEL AVIV

ISRAEL 69978

e-mail: aaklein@math.tau.ac.il 